\title{
QUALIDADE E VIDA ÚTIL PÓS-COLHEITA DE MELANCIA CRIMSON SWEET, COMERCIALIZADA EM MOSSORÓ
}

\author{
Sebastião Elviro de Araújo Neto ${ }^{1}$, Oscar Mariano Hafle², Fábio de Lima Gurgel³ \\ Josivan Barbosa Menezes ${ }^{4}$ \& Geomar Galdino da Silva ${ }^{5}$
}

\begin{abstract}
RESUMO
O objetivo desta pesquisa foi avaliar a qualidade e a vida útil pós-colheita de melancia cv. Crimson Sweet, comercializada em Mossoró, RN. A qualidade dos frutos foi avaliada por local de venda e tamanho de fruto. As melancias de tamanho grande e médio apresentaram melhor aparência externa e maior conteúdo de sólidos solúveis que aquelas de tamanho pequeno, enquanto as comercializadas na ocasião do desembarque apresentaram melhor aparência e maior firmeza. A avaliação da vida útil pós-colheita foi feita com frutos provenientes de um plantio comercial instalado em Mossoró, RN. A perda de peso foi de apenas 3,79\%, o pH da polpa aumentou de 4,89 para 5,20, a acidez total titulável decresceu durante o armazenamento e os conteúdos de sólidos solúveis e açúcares solúveis totais não apresentaram correlação com o armazenamento, sendo que o primeiro variou de 7,63 a 9,55\%. Os açúcares redutores apresentaram leve diminuição no final do armazenamento. A vida útil pós-colheita dos frutos foi avaliada em 12 dias.
\end{abstract}

Palavras-chave: Citrullus lanatus, qualidade, conservação, armazenamento

\section{QUALITY AND POSTHARVEST SHELF LIFE OF CRIMSON SWEET WATERMELON MARKETED IN MOSSORÓ}

\begin{abstract}
The objective of this research was to evaluate the quality and shelf life of Crimson Sweet watermelon marketed in Mossoró-RN. The quality of the fruits was evaluated by locality and fruit size. The large and middle sized watermelons presented better external appearance and higher soluble solids content than those of small size, while those marketed directly upon off loading, presented better appearance and higher firmness. The evaluation of the postharvest shelf life was done with fruits of a commercial plantation in Mossoró-RN. The weight loss was of $3.79 \%$, the $\mathrm{pH}$ of the fruits increased from 4.89 to 5.20 , the total titrable acidity decreased during storage. The soluble solids content and total soluble sugars did not present correlation with the storage, and the first varied from 7.63 to $9.55 \%$. The reducing sugars presented a slight decrease at the end of the storage. The postharvest shelf life of the fruits was evaluated at 12 days.
\end{abstract}

Key words: Citrullus lanatus, quality, shelf life, storage

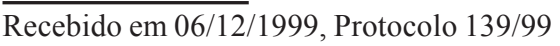

${ }^{1}$ Eng. Agr. M. Sc. em Fitotecnia - Universidade Federal do Acre - UFAC, Departamento de Ciências Agrárias BR 364 - km 04 - Campus Universitário. CEP 69915 - 900, Rio Branco, AC. E-mail: selviro@zipmail.com.br

${ }^{2}$ Prof. M.Sc. em Fitotecnia - Escola Agrotécnica Federal de Sousa, PB. E-mail: hafleom@zipmail.com.br

${ }^{3}$ Eng. Agr. M.Sc. em Fitotecnia - Escola Superior de Agricultura de Mossoró - ESAM. CP 137. CEP 59625 - 900, Mossoró, RN. E-mail: flgurgel@hotmail.com.br

${ }^{4}$ Eng. Agr. D.Sc. Pós-colheita - Escola Superior de Agricultura de Mossoró - ESAM. Fone: (0xx68) 312 2100. E-mail: quimica@esam.br

${ }^{5}$ Eng. Agr. M. Sc. Fitotecnia - ESAM 


\section{INTRODUÇ̃̃O}

A melancia é cultivada em muitos países do mundo, como: Índia, Irã, Itália e Estados Unidos. No Brasil, os estados de São Paulo, Goiás, Pernambuco e Bahia, são os maiores produtores, enquanto no Nordeste, além dos Estados citados, a região do Agropolo Mossoró - Assu, no Rio Grande do Norte, tem-se destacado pela sua crescente produção (Empasc, 1987; Araújo, 1989; Robinson \& Decker-Wolters, 1997). A comercialização da melancia é geralmente feita em condições ambiente, com vida útil menor comparadas àquelas melancias armazenadas $\mathrm{e}$ transportadas sob refrigeração (Risse et al., 1990).

$\mathrm{O}$ fruto da cv. Crimson Sweet apresenta formato arredondado, casca clara com estrias verde-escuro e polpa vermelho intenso muito doce, porém o fruto apresenta vida útil pós-colheita relativamente curta, principalmente quando não é acondicionado de forma adequada, afetando sua qualidade, uma vez que é consumido principalmente na forma in natura.

As principais variáveis usadas para definir a qualidade da melancia, são: conteúdo de açúcar, firmeza da polpa, sólidos solúveis (SS), aparência externa e interna e acidez total titulável (Elmostrom \& Davis, 1981; Brown \& Summers, 1985).

O conteúdo de açúcares varia de acordo com a região; nas variedades Charleston Gray e Klondike, por exemplo, o conteúdo de SS é maior na região central e menor na região próxima ao pedúnculo (Macgillivray, 1947; Chisholm \& Picha, 1986a). A concentração de SS decresce com o aumento da temperatura de armazenamento (Chisholm \& Picha, 1986b; Risse et al., 1990).

A frutose é o principal açúcar da melancia. O acúmulo de açúcar é observado de 20 a 36 dias após a antese. O conteúdo de frutose e glicose tende a diminuir após 28 dias a partir da antese, enquanto o conteúdo de sacarose e açúcares totais pode aumentar no período de 20 a 60 dias após a antese, dependendo da variedade (Elmostrom \& Davis, 1981; Brown \& Summers, 1985); quanto à firmeza da polpa, varia com a temperatura de armazenagem, embora algumas variedades mantenham sua firmeza, mesmo armazenadas com variação de temperaturas de $1^{\circ} \mathrm{C}$ inicialmente até $21^{\circ} \mathrm{C}$, no término do armazenamento, outras variedades tendem a diminuir (Risse et al., 1990).

$\mathrm{O}$ armazenamento prolongado, a baixas temperaturas torna as melancias sujeitas a várias formas de injúria por chilling, e em altas temperaturas ficam sujeitas à deterioração (Risse \& Hatton, 1982).

A melancia é uma das principais frutas comercializadas na Central de Abastecimento da CONAB (Companhia Nacional de Abastecimento), em Mossoró, cujo preço de venda é regulado de acordo com o seu tamanho, porém não se conhece a qualidade da melancia em relação ao tamanho. Associadas a isto, as condições de armazenamento não mantêm a qualidade do produto, implicando negativamente no preço de venda e na lucratividade do negócio.

Como a identificação dos fatores pós-colheita que diminuem a qualidade e o valor comercial da melancia, pode aumentar a eficiência na comercialização mantendo a qualidade, diminuindo perdas e ampliando lucros. Além disso, a determinação da vida útil deste produto oferece maior garantia de qualidade e segurança na sua comercialização.

Com esta pesquisa, objetivou-se avaliar a qualidade e a vida útil pós-colheita de melancias cv. Crimson Sweet, comercializadas e produzidas em Mossoró, RN.

\section{MATERIAL E MÉTODOS}

\section{Qualidade comercial}

Para avaliar a qualidade dos frutos comercializados em Mossoró, estes foram obtidos em duas quitandas (1 e 2) e, no momento do desembarque, na CONAB. Os frutos foram classificados de acordo com o tamanho de comercialização, em pequenos $(4,39 \pm 0,85 \mathrm{~kg})$, médios $(6,94 \pm 0,77 \mathrm{~kg})$ e grandes $(9,78 \pm 1,02 \mathrm{~kg})$. Foram tomados, como padrão de qualidade, a aparência (externa e interna), a firmeza, o pH, a acidez total titulável (ATT), os sólidos solúveis (SS), os açúcares redutores, não redutores e solúveis totais. O delineamento experimental utilizado para esta etapa do trabalho foi o de blocos completos casualizados, com três repetições, sendo cada parcela composta por um fruto. Os blocos representaram os diferentes locais de venda e a análise dos dados experimentais foi realizada através do aplicativo computacional SPSS (Norusis, 1990). Para a comparação entre médias, adotaram-se as recomendações de Gomes (1990).

\section{Vida útil pós-colheita}

Para se determinar sua vida útil, os frutos foram obtidos de um plantio comercial localizado no município de Mossoró,RN, cujo clima é quente e seco, com precipitação pluviométrica anual de $423 \mathrm{~mm}$, temperatura média máxima de $33^{\circ} \mathrm{C}$ e média mínima de $23^{\circ} \mathrm{C}$. Os frutos foram colhidos maduros, com peso médio de $5,14 \pm 0,67 \mathrm{~kg}$, e armazenados a temperatura de 22 a $26^{\circ} \mathrm{C}$ e 52 a $60 \%$ U.R., sendo as avaliações realizadas a cada três dias contando do dia da colheita. As variáveis analisadas foram: perda de peso, aparência (externa e interna), firmeza, sólidos solúveis, acidez total titulável e açúcares redutores, não redutores e solúveis totais. $\mathrm{O}$ delineamento experimental utilizado nesta etapa foi o inteiramente casualizado, com três repetições de um fruto por parcela e a análise de regressão foi realizada através do software table curve de Jandel Scientific (1991).

\section{Metodologia para análise}

Calculou-se a perda de peso pela diferença entre os pesos inicial e final, com os resultados expressos em percentagem (\%). A avaliação da aparência externa e interna foi feita de acordo com a metodologia adaptada de Menezes et al. (1995) utilizando-se uma escala subjetiva com notas de 1 a 5 , considerando-se a ausência ou a presença de defeitos, para aparência externa, como murcha ou ataque fúngico $(1=$ fruto extremamente deteriorado; $2=$ severo; $3=$ médio $; 4=$ leve; $5=$ ausência de defeitos); a aparência interna foi avaliada pela incidência de colapso interno, sementes soltas e líquido na cavidade das sementes $(1=$ fruto extremamente deteriorado; 2 = severo; 3 = médio; 4 = leve; $\quad 5=$ ausência de danos), frutos com nota $\leq 3,0$ foram considerados de baixa qualidade para o consumo. Para avaliação da firmeza, o fruto foi dividido longitudinalmente, sendo que em cada uma das metades foram realizadas três leituras (na região equatorial do fruto, sendo uma no centro e duas nas laterais) usando-se penetrômetro manual com ponteira cilíndrica de $12 \mathrm{~mm}$ de diâmetro e os resultados obtidos em libra (Lbf) e expressos em newton (N) através do fator de conversão 4,445. O conteúdo de sólidos solúveis foi determinado, usando refratômetro digital com 
correção automática de temperatura, em amostras retiradas de uma fatia longitudinal da polpa do fruto homogeneizada em liquidificador, sendo os resultados expressos em percentagem. Os açúcares redutores e não redutores foram determinados no suco e expressos em percentagem. Os açúcares solúveis totais pela somatória dos dois primeiros e expressos em percentagem; o método utilizado foi o de Somoghy-Nelson (Southgate, 1991). A concentração total da amostra para leitura da absorbância foi de $1250 \mu \mathrm{L} \mathrm{mL}^{-1}$, para amostras não hidrolisadas e $750 \mu \mathrm{L}$ $\mathrm{mL}^{-1}$, para amostras hidrolisadas.

\section{RESULTADOS E DISCUSSÃO}

\section{Qualidade comercial}

$\mathrm{O}$ resultado dos parâmetros aparência interna, firmeza, $\mathrm{pH}$, e açúcares redutores, não redutores e totais, foi semelhante para os três tamanhos de fruto $(\mathrm{P} \leq 0,05)$. As melancias maiores e médias apresentaram aparência externa melhor que aquelas de tamanho pequeno (Tabela 1).

Com base nos dados deste experimento, Tabela 1, os frutos de melancia de tamanhos grande e médio apresentaram melhor qualidade quanto à doçura, pois o conteúdo de SS foi significativamente maior. Em melão, Cohen \& Hicks (1986) afirmam que o primeiro atributo percebido no flavor é a doçura, e quando o melão ocasionalmente tiver alto conteúdo de SS, este será considerado melão de flavor alto, explicando assim, a forte correlação encontrada entre SS vs aceitabilidade, doçura e flavor.

Os locais de venda comercializam melancias com diferentes qualidades comerciais, sendo as comercializadas diretamente no local de desembarque na CONAB, apresentam qualidade superior às das quitandas, com melhor aparência interna, mais firmeza e maior conteúdo de açúcares redutores.

Com base na aparência interna, firmeza da polpa e teor de açúcares redutores das melancias comercializadas logo após a colheita (desembarque, Tabela 1), e considerando-as como padrão de qualidade para esta cultivar produzida em Mossoró, pode afirmar-se que: melancias expostas em quitandas sob condição ambiente, são comercializadas em estádio de senescência, com baixa qualidade para consumo. O decréscimo na concentração de açúcares redutores pode ser explicado pela pouca reserva de amido no fruto e pela perda de monossacarídeos no processo respiratório, acelerado pelas altas temperaturas de armazenamento (Chisholm \& Picha, 1986b).

\section{Vida útil pós-colheita}

A perda de peso ao final do experimento (21 dias) foi de $3,79 \%$, não causando murchamento nos frutos, que mantiveram boa aparência comercial; portanto, a melancia Crimson Sweet apresenta boa resistência à perda de peso, durante o armazenamento sob condição ambiente (Figura 1).

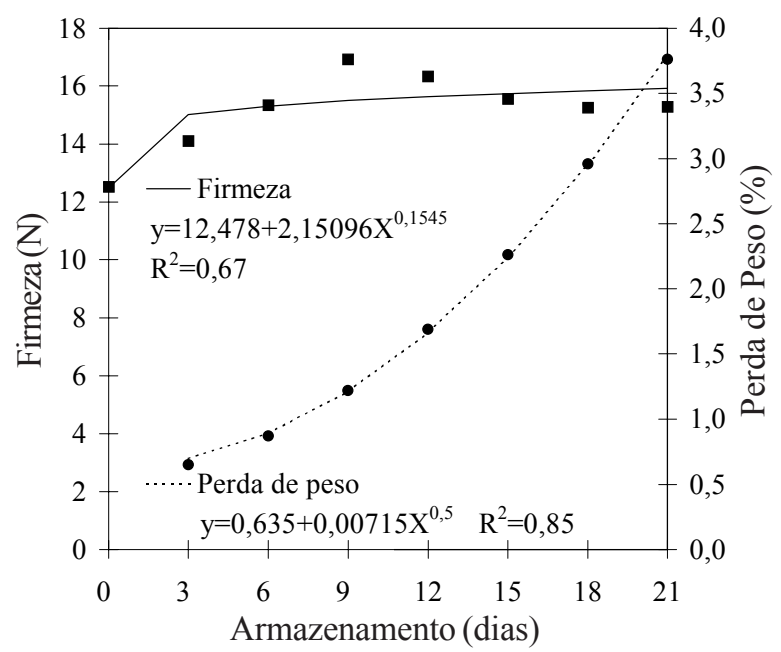

Figura 1. Firmeza e perda de peso de melancias armazenadas sob condição ambiente, com temperaturas de 22 a $26^{\circ} \mathrm{C}$ e 52 a $60 \%$ de U.R.

A firmeza da polpa teve aumento a partir do $3^{\circ}$ dia de armazenamento (Figura 1), permanecendo constante até o final do experimento, semelhante ao comportamento observado em outros trabalhos com melancia (Risse et al., 1990). Este comportamento está relacionado a uma elasticidade da polpa (observação gustativa) que reduziu o aspecto crocante, característico da textura de melancia, limitando a vida útil pós-colheita desses frutos em apenas 12 dias, quando armazenados nestas condições.

A aparência externa e/ou interna da melancia não foi fator limitante na vida útil desses frutos (Figura 2) pois, aos 18 dias de armazenamento, estes ainda se apresentavam com boa

Tabela 1. Características físicas e químicas" de melancia cv. Crimson Sweet, de diferentes tamanhos e locais de venda, comercializada na cidade de Mossoró, RN, avaliada pela aparência externa e interna (notas de 1 a 5), firmeza, pH, acidez (ATT), sólidos solúveis (SS), açúcares redutores (AR), açúcares não redutor (ANR) e açúcares solúveis totais (AT)

\begin{tabular}{|c|c|c|c|c|c|c|c|c|c|}
\hline \multirow[t]{2}{*}{ Tratamento } & \multicolumn{2}{|c|}{ Aparência } & \multirow{2}{*}{$\frac{\text { Firmeza }}{(\mathrm{N})}$} & \multirow[t]{2}{*}{$\mathrm{pH}$} & \multirow{2}{*}{$\begin{array}{c}\text { Acidex } \\
\mathrm{mmol} \mathrm{H}^{+} 100 \mathrm{~mL}^{-1}\end{array}$} & SS & $\overline{\mathrm{AR}}$ & ANR & $\mathrm{AT}$ \\
\hline & Externa & $\overline{\text { Interma }}$ & & & & \multicolumn{4}{|c|}{$\%$} \\
\hline \multicolumn{10}{|l|}{ Tamanho } \\
\hline Pequeno & $3,2 \mathrm{~b}$ & $3,5 \mathrm{a}$ & $13,20 \mathrm{a}$ & $5,40 \mathrm{a}$ & $1,98 \mathrm{~b}$ & $8,46 \mathrm{~b}$ & $8,46 \mathrm{~b}$ & $1,27 \mathrm{a}$ & $6,37 \mathrm{a}$ \\
\hline Médio & $3,6 \mathrm{ab}$ & $4,0 \mathrm{a}$ & $12,43 \mathrm{a}$ & $5,47 \mathrm{a}$ & $2,30 \mathrm{ab}$ & $9,98 \mathrm{a}$ & $5,41 \mathrm{a}$ & $1,93 \mathrm{a}$ & $7,34 \mathrm{a}$ \\
\hline Grande & $4,1 \mathrm{a}$ & $4,3 \mathrm{a}$ & $12,58 \mathrm{a}$ & $5,48 \mathrm{a}$ & $2,50 \mathrm{a}$ & $10,06 \mathrm{a}$ & $5,12 \mathrm{a}$ & $1,52 \mathrm{a}$ & $6,64 \mathrm{a}$ \\
\hline \multicolumn{10}{|l|}{ Local } \\
\hline Quitanda 1 & $3,2 \mathrm{~b}$ & $3,5 \mathrm{~b}$ & $12,15 \mathrm{~b}$ & $5,56 \mathrm{a}$ & $1,93 \mathrm{~b}$ & $8,64 \mathrm{a}$ & $4,84 \mathrm{~b}$ & $0,88 \mathrm{~b}$ & $5,74 \mathrm{~b}$ \\
\hline Quitanda 2 & $3,4 \mathrm{ab}$ & $3,7 \mathrm{~b}$ & $9,23 \mathrm{c}$ & $5,44 \mathrm{ab}$ & $2,44 \mathrm{ab}$ & $10,11 \mathrm{a}$ & $4,32 \mathrm{~b}$ & $2,81 \mathrm{a}$ & $7,13 \mathrm{a}$ \\
\hline Desembarque & $4,3 \mathrm{a}$ & $4,7 \mathrm{a}$ & $16,83 \mathrm{a}$ & $5,33 \mathrm{~b}$ & $2,35 \mathrm{a}$ & $9,75 \mathrm{a}$ & $6,47 \mathrm{a}$ & $1,02 \mathrm{~b}$ & $7,50 \mathrm{a}$ \\
\hline Desv. Padrão & 0,8 & 0,9 & 3,85 & 0,18 & 0,42 & 1,42 & 1,38 & 1,44 & 1,37 \\
\hline C.V. (\%) & 22,2 & 22,7 & 30,23 & 3,30 & 18,73 & 14,95 & 26,5 & 91,5 & 23,7 \\
\hline
\end{tabular}


aparência externa e interna, com nota acima de 3 , considerada comercial; o pH dos frutos aumentou de 4,89 a 5,20 durante o período de armazenamento (Figura 3). Estudando o melão "Makdimon", Wang et al. (1996) observaram aumento do pH, de 5,3 para 6,75 , e que essas mudanças têm forte correlação com a diminuição do conteúdo dos ácidos orgânicos e com as mudanças em SS e açúcares solúveis totais.

A acidez total titulável decresceu 58,6\% durante o armazenamento (Figura 3); resultados semelhantes foram encontrados por Menezes et al. (1995), em melão amarelo "Agroflora", com redução de $35,5 \%$ aos 45 dias de armazenamento e redução de $41,6 \%$ em melão analisado por Gonçalves et al. (1996).

O conteúdo de sólidos solúveis não apresentou correlação com o armazenamento e variou de 7,63 a 9,55\% (Figura 4), sendo que, no final do experimento houve tendência de diminuição, devido a melancia, após colhida, não possuir reserva de carboidratos, como o amido, para ser convertido em açúcar, e os açúcares presentes no momento da colheita serem utilizados no processo respiratório (Pratt, 1971; Seymour et al., 1993).

O conteúdo de açúcares solúveis totais, redutores e sólidos solúveis, apresentaram o mesmo comportamento bioquímico, com tendência a redução durante o armazenamento (Figura 5).

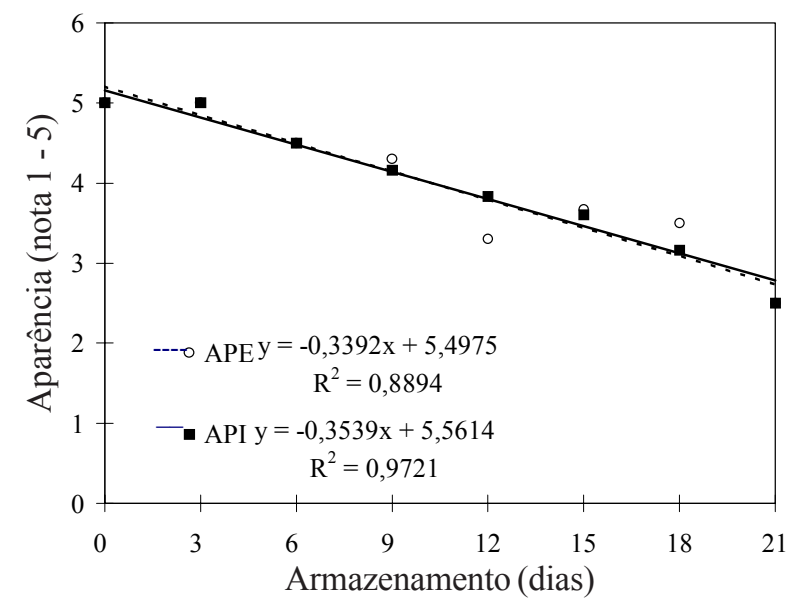

Figura 2. Aparência externa (APE) e aparência interna (API) de melancias armazenadas sob condição ambiente, com temperatura de 22 a $26{ }^{\circ} \mathrm{C}$ e 52 a $60 \%$ de U.R.

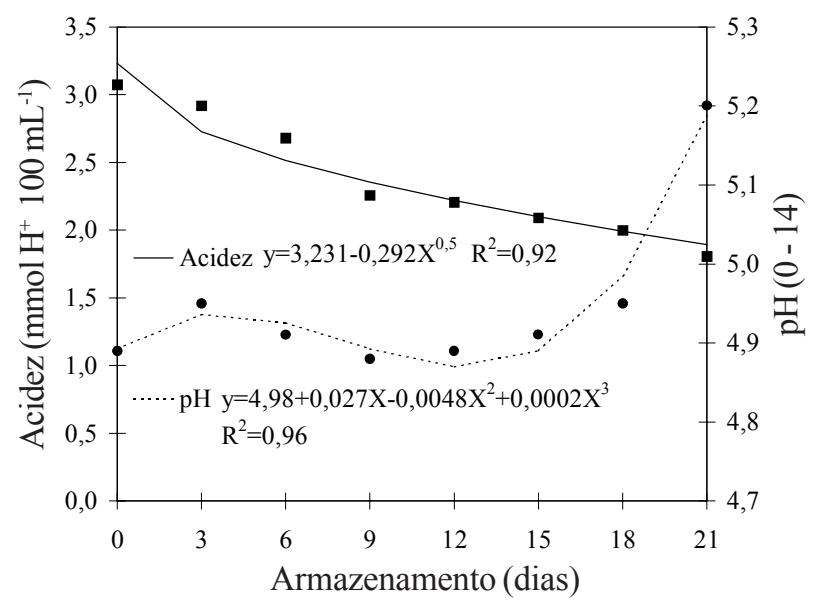

Figura 3. Acidez total titulável e $\mathrm{pH}$ de melancias armazenadas sob condição ambiente, com temperatura de 22 a $26{ }^{\circ} \mathrm{C}$ e 52 a $60 \%$ de U.R.

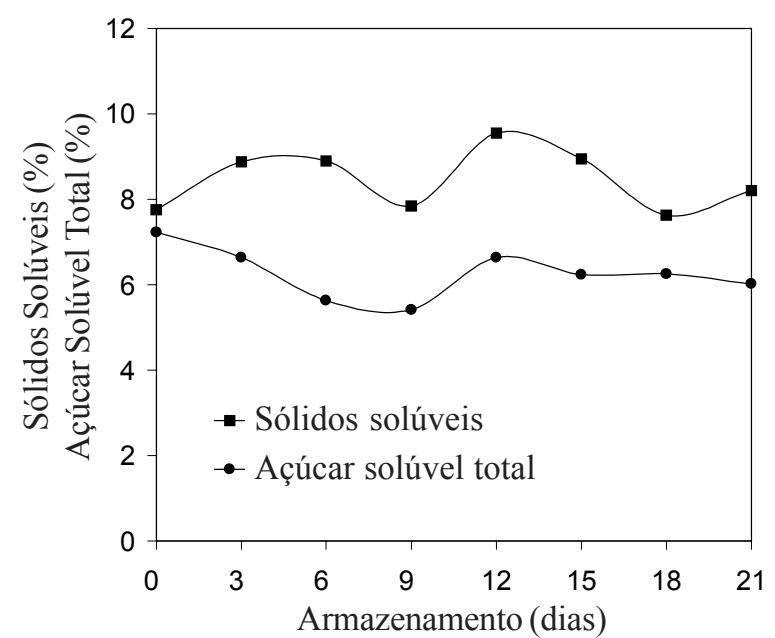

Figura 4. Sólidos solúveis e açúcares solúveis totais de melancias armazenadas sob condição ambiente, com temperatura entre 22 e $26^{\circ} \mathrm{C}$ e 52 e $60 \%$ de U.R.

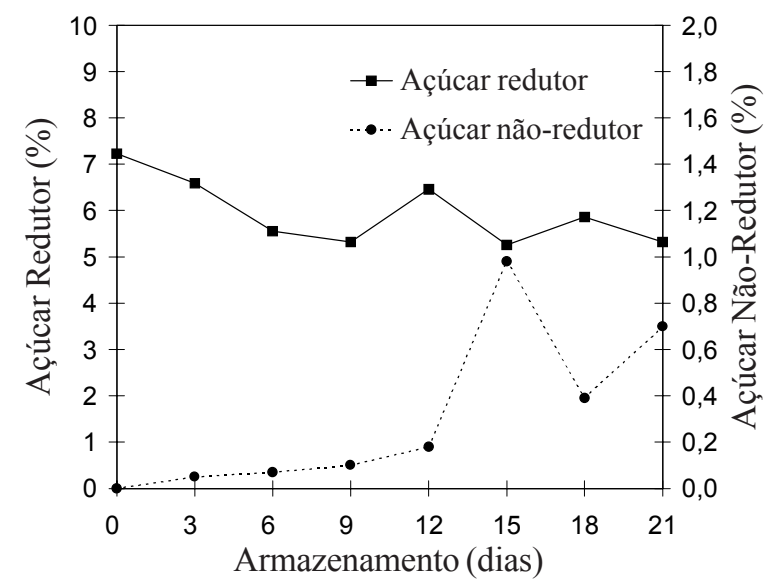

Figura 5. Açúcares redutor e não redutor de melancias armazenadas sob condição ambiente, com temperaturas de 22 a $26^{\circ} \mathrm{C}$ e 52 a $60 \%$ de U.R.

Os açúcares não-redutores foram encontrados em baixas quantidades durante todos os estágio do armazenamento (Figura 5), cujos resultados confirmam aqueles encontrados em outros experimentos (Chisholm \& Picha, 1986b; Risse et al.,1990).

\section{CONCLUSÕES}

1. A qualidade de frutos de melancia da variedade Crimson Sweet é influenciada pelo tamanho do fruto, sendo que a melhor qualidade foi encontrada em frutos médios e grandes.

2. A qualidade da melancia varia com o local de comercialização.

3. A vida útil pós-colheita da melancia da variedade Crimson Sweet, acondicionada em condição ambiente de Mossoró, é de apenas 12 dias.

\section{REFERÊNCIAS BIBLIOGRÁFICAS}

ARAÚJO, J.P. de. A cultura da melancia (Citrullus lanatus). Petrolina: Embrapa-CPATSA, 1989. 9p. Embrapa-CPATSA, Comunicado Técnico, 35 
BROWN Jr, A.C.; SUMMERS, W.L. Carbohydrate accumulation and color development in watermelon. Journal of the American Society for Horticultural Science, Mount Vernon, v.110, n.5, p.683-687, 1985.

CHISHOLM, D.N; PICHA, D.H. Distribution of sugar and organic acids within ripe watermelon fruit. HortScience, Alexandria, v.21, n.3, p.501-503, 1986a.

CHISHOLM, D.N; PICHA, D.H. Effect of storage temperature on sugar and organic acid contents of watermelon. HortScience, Alexandria, v.21, n.4, p.1031-1033, 1986 b.

COHEN. R.A; HICKS, J.R. Effect of storage on quality and sugars in muskmelon. Journal of the American Socienty of Horticultural Science, Mount Vernon, v.111, n.4, p.553-557, 1986.

ELMOSTROM, G.W.; DAVIS, P.L. Sugar in developing and mature fruits of several watermelon cultivars. Journal of the American Society of Horticultural Science, Mount Vernon, VA, v. 106, n.3, p.330-333, 1981.

EMPASC (Florianópolis, SC). Normas técnicas para a cultura da melancia: Região do litoral de Itajaí. Florianópolis: EMATERSCACARESC, 1987. 18p. EMPASC. Sistema de Produção, 8

GOMES, F.P. Curso de estatística experimental. Piracicaba: Nobel, 1990. 250p.

GONÇALVES, F. das C.; MENEZES, J.B.; ALVES, R.E. Vida útil pós-colheita de melão "Piel de Sapo" armazenado em condições ambiente. Horticultura Brasileira, Brasília, DF, v.14, n.1, p.49-52, 1996.

JANDEL SCIENTIFIC. User's Manual. California: Jandel Scientific, 1991.280p.
MENEZES, J.B.; CHITARRA, A.I.F.; CHITARRA, M.A. de; CARVALHO, H.A. de. Caracterização pós-colheita do melão amarelo “Agroflora 646”. Horticultura Brasileira, Brasília, DF, v.13, n.2, p.150-153, 1995.

MACGILLIVRAY, J.H. Soluble solids content of diferent regions of watermelons. Plant Physiology, Bethesda, Md, v.22, p.637-640, 1947.

NORUSIS, M. J. SPSS statistics. Illinois: SPSS Inc, 1990.

PRATT, H.K. Melões. In: HULME, A.C. Biochemistry of fruits and their products. New York: Academic Press, 1971. p.207-323.

RISSE, L.A.; BRECHT, J.K.; SARGENT, S.A.; LOCASCIO, S.J.; CRALL, J.M.; ELMSTROM, G.W.; MAYANRD, D.N. Storage characteristic of small watermelon cultivars. Journal of the American Society for Horticultural Science, Mount Vernon, v.115, n.3, p.440-443, 1990.

RISSE, L.A.; HATTON, T.T. Sensitivity of watermelons to ethylene during storage. HortScience, Alexandria, v.6, n.17, p.946-048. 1982.

ROBINSON, R.W.; DECKER-WOLTERS, D.S. Cucurbits. Waflingford: CAB International, 1997. 226p.

SEYMOUR, G.B.; TAYLOR, J.E.; TUCKER, G.A. Biochemistry of fruit ripening. London: Chapman \& Hall, 1993. 454p.

SOUTHGATE, D.A.T. Determination of foods carbohydrates. London: Applied Science, 1991. 232p.

WANG, Y.; WYLLIE, S.G.; LEACH, D.N. Chemical changes during the development and ripening of the fruit of Cucumis melo (Cv. Makdimon). Journal Agricultural Food Chemistry, Washington, DC, v. 44, p.210-216, 1996. 